\title{
A Mechanism for the effects of Relativity
}

\author{
Remi Cornwall
}

Future Energy Research Group

Queen Mary, University of London, Mile End Road, London E1 4NS

http://webspace.qmul.ac.uk/rocornwall http://www.cornwallresearch.org

\begin{abstract}
This paper seeks to investigate the questions of: How does the constancy of the speed of light come about? Why does time dilation and length contraction occur? Are they physical effects with a mechanism? Does mass have a role is in these effects? Is Relativity an emergent phenomenon? The enquiry is along a different tact than the standard Lorentzian invariance canon but in the realm of readily known experimental facts or analogies and theory in the domain of wave propagation and solid state physics. These analogies, to almost prosaic physics, have a small following and are called Ether Theories, which modern physics has implicitly reinstated by General Relativity and Quantum Field Theories. In the category of Ether Theories based on analogies to solid state physics, this presentation is unique in not being Lorentz invariant; it is based on earlier papers by the author enquiring into the speed of coincidence counting of the Bell Inequality and a communication protocol. It is believed that Lorentz invariance emerges from the Ether and all Relativistic Mechanics can be built from the bottom up. The conclusion is that space-time is not really curved but the effects are all ascribable to mass gain.
\end{abstract}

Keywords: Relativity, EPR, Superluminal, Ether, Sudarshan, Effective mass, time dilation, length contraction

\section{Introduction I}

The speed of light occurs in many places in physics, most obviously in electrodynamics but also in fundamental physics. The peculiar way it enters Maxwell's electrodynamics and the wave equation was noted from the outset as not obeying Galilean Relativity. Yet the founders of electrodynamics, Faraday, Gauss, Ampere (et al) and Maxwell too[1-3] always thought in terms of a physical medium through which electromagnetic phenomena, especially waves, propagated.

The Michelson-Morely experiment seemed to dispel notions of a mechanical ether because it would have been expected that variations in the speed of light should be detected by the passage of the source or observer through the medium.

Fantastical ideas were put about at the time: "ether drag", "time dilation" (Lorentz, Poincare), "length contraction" (Fitzgerald). We have come to accept the latter two when Einstein (and contemporaneously, Poincare) developed Special Relativity and boldly took the constancy of the speed of light as an axiom with the concomitant time dilation and length contraction as necessary. These intriguing phenomena have been directly and "indirectly" (by inference) confirmed respectively by atomic clocks[1] or the detection of muons at sea level from cosmic radiation[1] and synchrotron radiation[1] to cite a few examples.

The core feature of Special Relativity is two axioms: The Constancy of the Speed of Light and the Principle of Relativity. Moving on from inertial frames Einstein then took as core other principles, that all motion looks most simple in free-fall and the Equivalence Principle to formulate General Relativity. The upshot of this theory is still one of length contraction and time dilation but with additional effect caused by the gravitational field (which is now called the metric tensor and related to the stress energy tensor).

Locally (or hence in an infinitesimal region) light speed is still constant, however light is observed to bend or "lens" around gravitating bodies and the Shapiro delay[4] shows that, compared to flat space, light speed is slowed. This seems to suggest that space has dielectric like properties[4] and since there is "nothing there" apart from empty space, it must be, from the viewpoint of applied physicists or even electrical engineers, perfused with a medium.

Mathematical physicists, in the legerdemain of esoteric mathematics, never really seem to acknowledge or even want to admit the physical substance of space; they speak in abstractions of curved space-time. Yet in another area of physics, Quantum Field Theories, they contend with physical matter popping out of the vacuum. They struggle with yet more mathematics trying to link these two areas of physics and descend into realms of non-science, that is, metaphysics and that which cannot even be directly inferred from experiment (multi-dimensional space-time, "multiverses" and other such fashions).

There is no doubt that the abstraction provided by mathematical physics is powerful, great successes have been delivered by it for over 300 years. Yet it seems to forget that physics is not "mere" mathematics and a symbiotic relationship exists between mathematics, pure physics, applied physics and engineering. It is not only that applied physicists and engineers have well founded intuitions based on physical modelling but the technological needs of society requires engineering in new realms traditionally the preserve of the 
mathematical theorists. It is not just the level of abstraction which is off putting but taking on faith things which can never be truly measured or the use of mind-bending concepts, when Occam's Razor/The Principle of Parsimony would suggest an easier one. We believe that those of a more applied or engineering mindset have just as valid a contribution to make to "fundamental" physics, as the supposed intuition of the overly mathematically inclined - with no Faraday, there would have been no Maxwell equations.

\section{Introduction II - The case for Ether Theories}

Our starting point is the concepts surrounding the effective mass familiar to applied physicists or material scientists and semiconductor engineers and also the dispersion relations familiar to waveguide engineers:

A general sinusoidal plane wave propagating is given by:

$$
A(\mathbf{r}, t)=A e^{i(\omega t \pm \mathbf{k} \cdot \mathbf{r})}
$$

Or a spherical wave by:

$$
A(r, t)=\frac{A}{r} e^{i(\omega t \pm k r)}
$$

Where $\omega$, is the angular frequency which specifies the number of cycles (radians) per unit time and $\mathrm{k}$, is the wavenumber, which is the number of cycles (radians) per unit distance.

Standard wave theory[3] gives the phase velocity as:

$$
v_{\text {phase }}=\frac{\omega}{k}
$$

A particle is associated with a "wavepacket"[5-7] of interfering waves close in frequency. The movement of this packet is associated with the group velocity:

$$
v_{\text {group }}=\frac{\partial \omega}{\partial k}
$$

The frequency in a medium is in general a function of the wavenumber, $\omega(\mathrm{k})$, thus so are both the phase and group velocities. Such a medium is called dispersive. As a quick and well known example in optics, take the refractive index:

$$
n=\frac{c}{v_{\text {phase }}}
$$

The group velocity is then:

$$
\frac{d \omega}{d k}=\frac{c}{n}-\frac{c k}{n^{2}} \frac{d n}{d k}
$$

And the group velocity will only be the same as the phase velocity when the refractive index is constant. Thus in empty space, all frequencies of light travel at the same speed.

One of the most profound unifications in physics came from Quantum Theory and wave-particle duality; familiar concepts from the domains of Electromagnetics were compared and contrasted with ideas in Mechanics. The Planck's analysis of the black-body spectrum and Einstein's analysis of the photo-electric effect lead to the energyfrequency relation[1]:

$$
E=\hbar \omega
$$
eqn. 6

Special Relativity offered another profound unification, of matter and energy. Energy and momentum formed a 4-vector, as well frequency and wavenumber. By this reasoning de Broglie deduced another relation:

$$
\mathbf{p}=\hbar \mathbf{k}
$$

Observation of electron diffraction said that matter has a wave like nature too and the above relations could be generally applicable. Schrödinger, seeing the similarities between the solution to the electromagnetic wave equation in operator form and its solutions, was then able to deduce the operator forms of energy and momentum that would act on the "wavefunction":

$$
\begin{aligned}
& \text { if } \nabla^{2} \psi-\frac{1}{c^{2}} \frac{\partial^{2} \psi}{\partial t^{2}}=0 \text { and } \rightarrow \psi=\psi_{0} e^{i(w t \pm \mathbf{k} \cdot \mathbf{r})} \\
& \text { if } \frac{\partial \psi}{\partial x}= \pm i k_{x} e^{i(w t \pm \mathbf{k} \cdot \mathbf{r})}= \pm k_{x} \psi
\end{aligned}
$$

Then by eqn. 7 we can write:

$$
\hat{\mathbf{p}}=-i \hbar \nabla
$$

And similarly by eqn. 6 that,

$$
\hat{E}=i \hbar \frac{\partial}{\partial t}
$$

The history of science tells us[1] that, the Schrödinger Equation was arrived at after a failed attempt to have solutions to a relativistically correct matter-wave equation, based on the known relativistically correct wave equation from Electrodynamics or the Klein-Gordon equation:- 
From the invariant squared magnitude of the energy-momentum four vector:

$$
E^{2}-(\mathbf{p} c)^{2}=\left(m c^{2}\right)^{2} \quad \text { eqn. } 10
$$

In operator form we arrive immediately at the Klein-Gordon equation:

$$
\begin{gathered}
\hat{E}^{2}-(\hat{\mathbf{p}} c)^{2}=\left(m c^{2}\right)^{2} \\
\Rightarrow \frac{\partial^{2} \psi}{\partial t^{2}}-c^{2} \nabla^{2} \psi=\left(\frac{m c^{2}}{\hbar}\right)^{2} \psi
\end{gathered}
$$

Schrödinger settled on the operator analogue to the classical Hamiltonian (the total energy of the system) and was able to model the hydrogen atom. This confirmed the general applicability of quantum mechanics (even though its inception was regarding electromagnetic phenomena), the de Broglie relations, wave-particle and energymomentum duality/unification.

$$
\text { if } H=\frac{p^{2}}{2 m}+V(r)
$$

By analogy for matter waves he wrote:

$$
\begin{gathered}
\hat{H} \psi=i \hbar \frac{\partial}{\partial t} \psi \\
\Rightarrow-\frac{\hbar^{2}}{2 m} \nabla^{2} \psi+V \psi=i \hbar \frac{\partial}{\partial t} \psi
\end{gathered}
$$

Considering the phase velocity (eqn. 4 ) and the de Broglie relations, we can write:

$$
v_{\text {group }}=\frac{\partial E}{\partial \mathbf{p}}
$$

And note non-Relativistically:

$$
v_{\text {group }}=\frac{\partial}{\partial p}\left(\frac{1}{2} \frac{p^{2}}{m}\right)=\frac{p}{m}=v
$$

Or Relativistically (from eqn. 10):

$$
\begin{aligned}
& v_{\text {group }}=\frac{\partial}{\partial p} \sqrt{p^{2} c^{2}+m^{2} c^{4}} \\
& =\frac{p c^{2}}{\sqrt{p^{2} c^{2}+m^{2} c^{4}}}
\end{aligned}
$$

Which after a little re-arrangement and substitution of the relativistic momentum:

$$
\mathbf{p}=\frac{m \mathbf{v}}{\sqrt{1-\frac{v^{2}}{c^{2}}}}
$$

We find,

$$
v_{\text {group }}=\frac{p}{m \sqrt{1-\frac{v^{2}}{c^{2}}}}=v
$$

Thus, just as for the case of electromagnetic waves, the quantum wave equations and the de Broglie relations correspond to the movement of energy by a localised wavepacket, which is similar to a classical particle viewpoint (ie. Quantum Mechanics must also govern Electrodynamics too). Furthermore, by a similar Correspondence Principle with the old ideas of Classical Mechanics, if we can write:

$$
\frac{d p}{d t}=m \frac{d v}{d t} \quad \Rightarrow \frac{\hbar d k}{d t}=m \frac{d}{d t}\left(\frac{d E}{d p}\right)
$$

Then,

$$
\begin{aligned}
& \frac{\hbar d k}{d t}=m \frac{d}{d t}\left(\frac{1}{\hbar} \frac{d E}{d k}\right)=\frac{m}{\hbar} \frac{d^{2} E}{d k^{2}} \frac{d k}{d t} \\
& \Rightarrow m=\frac{\hbar^{2}}{\frac{d^{2} E}{d k^{2}}} \quad \text { or } \quad m=\frac{1}{\frac{d^{2} E}{d p^{2}}}
\end{aligned}
$$

So far our consideration has been to only one particle (or wavepacket) in free space. In bulk material we could contend with the solution of the multi-particle form of eqn. 12 with all their interacting potentials. However, focusing on one particle of interest and using a mean-field approach to the interaction of the other particles, applied physicists, material scientists and electronic engineers working with semiconductors use eqn. 17 as the "effective mass", of say, an electron in a lattice which otherwise behaves as if it is in free space, but with a modified mass.

Here now is the crux of the Ether Theory argument: Is what we call "free space" really a plenum with material properties such that a particle has a "bare" mass (or even non-existent mass) which has arisen from its interaction with that plenum? Might it be that the Schrödinger equation is really this? :-

$$
\begin{gathered}
\underbrace{-\frac{\hbar^{2}}{2 m} \nabla^{2} \psi}_{\downarrow}+V \psi=i \hbar \frac{\partial}{\partial t} \psi \\
\left(-\frac{\hbar^{2}}{2 \varepsilon} \nabla^{2} \psi+V_{\text {ether }} \psi\right)+V \psi=i \hbar \frac{\partial}{\partial t} \psi \text { eqn. } 18
\end{gathered}
$$

Where the constant $\varepsilon$ is the bare mass that vanishes to zero but the interaction with the Ether field 
creates the "effective mass" we call "mass" ${ }^{\dagger}$. Also the bracketed term in eqn. 18 is the kinetic energy and we could now regard the velocity (squared in eqn. 14 or a function of it in eqn. 16) as a "displacement" in the ether field, putting kinetic energy on the same footing as potential energy.

It is interesting to note too that even in the relativistic regime, eqn. 13, the de Broglie relations and the wave kinematics it is built upon still hold. This suggests that wave mechanics is even more fundamental than Relativity. This will be taken up in the next section where both Special and General Relativity are seen to be emergent phenomena from an underlying Ether Theory.

\section{The Author's departure point}

Modern thought on an Ether, in the light of the Quantum Mechanics and Relativity revolutions, was started by Dirac and his Dirac Sea, which was a philosophical attempt to understand the negative solutions of the Dirac Equation[1, 8]. In the discipline of General Relativity, Sakharov[4] proposed that gravity was the "metric elasticity" of space associated with quantum fluctuations of other fields. Sudarshan et al[9] believed the ether to be composed of fermion/anti-fermion pairs and used concepts from condensed matter physics, such as superfluidity and band theory to show that all particles were an excitation of this ether and account for their rest masses as the energy of excitation[10].

Unlike Sudarshan we take the physics of the multiple particle fields as a given and no unification of these is being attempted. In this paper, there is one mission and methodology: to explain how Relativistic effects and Lorentz invariance come about and the finite speed of light, to build Relativity from a more fundamental starting point by looking at mass.

We believe that there is an unique starting point coming from the author's earlier thoughts[11-12] on the speed of coincidence counting in Bell Inequality tests[13], in which the author stripped down the Lorentz transform by removing the retarded time terms from it (figure 1), such that an absolute reference frame at absolute stillness could have a transform to view the rest of the Universe. There now follows a quick re-cap of the findings. In its most simple form the transform found was:

$$
\begin{aligned}
& T=\gamma t \\
& X=\gamma x
\end{aligned}
$$$$
\text { eqn. } 19
$$

$\uparrow$ The bracketed term in eqn. 18 is the kinetic energy and we could replace $V_{E t h e r} \psi$ with an operator as a function of

$\frac{\partial E}{\partial \mathbf{p}}$ acting on the wavefunction to recover the usual $-\frac{\hbar^{2}}{2 m} \nabla^{2} \psi$

$$
\begin{aligned}
& \text { The Lorentz transform: } x=\gamma\left(x^{\prime}+v t^{\prime}\right) t=\gamma\left(t^{\prime}+\frac{v x^{\prime}}{c^{2}}\right) \\
& \begin{array}{lrrrr}
u=\left(x_{1} x_{2} x_{3} \text { ict }\right) & 1 & 0 & 0 & 0 \\
u^{\prime}=L(\alpha) u & 0 & 1 & 0 & 0
\end{array} \quad \text { Where } \alpha=\tanh ^{-1} \\
& u^{\prime}=L(\alpha) u \quad L=\begin{array}{cccc}
0 & 1 & 0 \\
0 & 0 & \cosh \alpha & i \sinh \alpha
\end{array} \\
& \begin{array}{llll}
0 & 0 & -i \sinh \alpha & \cosh \alpha
\end{array}
\end{aligned}
$$

Thus we obtain the familiar space-time diagram:

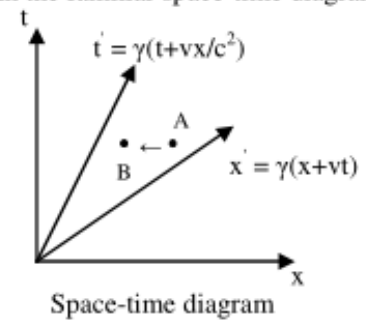

The terms in the Lorentz transform $\Delta \mathrm{x}=\gamma \mathrm{v} \Delta \mathrm{t}^{\prime}$ and

$\Delta \mathrm{t}=\gamma \mathrm{v} \Delta \mathrm{x}^{\prime} / \mathrm{c}^{2}$ can simply be understood as the delay in sending the information about the co-ordinates to the nonprimed frame. For instance if it takes the primed frame $\Delta \mathrm{t}^{\prime}$ seconds to perform a measurement then the frame will have moved a distance $v \Delta t^{\prime}$ which we correct back to the un-primed frame, $\gamma \mathrm{v} \Delta \mathrm{t}^{\prime}$ in addition to any other distance measurement. As regards the time: the frame will have moved $v \Delta t$ ' once again so the light signal will require an extra $v \Delta t^{\prime} / \mathrm{c}$ seconds to reach the source, now $\Delta \mathrm{t}^{\prime}=\Delta \mathrm{x}^{*} / \mathrm{c}$ so the extra time is $\gamma \mathrm{v} \Delta \mathrm{x}^{\prime} / \mathrm{c}^{2}$ in the un-primed frame.

Sending information superluminally knocks out the terms $\Delta \mathrm{x}=\gamma \mathrm{v} \Delta \mathrm{t}^{\prime}$ and $\Delta \mathrm{t}=\gamma \mathrm{v} \Delta \mathrm{x}^{\prime} / \mathrm{c}^{2}$ in the Lorentz transform giving the following transformation diagram:

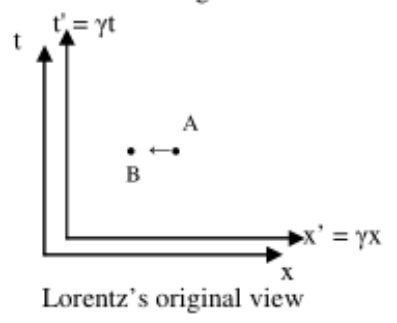

Figure 1 - Removal of the retarded time terms from the Lorentz Transform leads to Absolute Space and Time.

The thought underpinning this was a protocol for sending signals by entangled pairs developed by the author[11-12]; it is possible to infer measurement or non-measurement of entangled pairs[14] non-locally, which the author then realised could be used to send classical data down a quantum channel. Compared to a light-speed signal communicating the same data, the collapse of the 
distant wavefunction by the generation of a mixed state (which is discernable by an interferometer) is faster even instantaneous (a disproof of the No Communication Theorem is in[12]).

This means of sending data and comparing results against light signals, in a number of familiar experimental scenarios in Relativity theory (Doppler shift, length contraction etc.), lead the author to see that the Lorentz transform and invariance is a combination of length contraction, time dilation and the retarded time to send light speed signals; a privileged position in space at absolute rest, un-time dilated, un-length contracted communicating with the other bodies by superluminal signals, would discern measurements by eqn. 19. Light would travel at "c" in this frame too and that would be the speed limit for everything. Bodies travelling through space would have their clocks slow and dimensions contract, yet when bodies communicate with each other by faster than light speed signals (and indeed to and from the preferential reference frame too) they would discern the reciprocal Lorentz transform and that the other body has slowed and contracted.

Thus eqn. 19 broke the reciprocity of the Lorentz transform by superluminal signals so that bodies would know they were moving. The privileged rest frame would imbue physical properties to space to make all the other bodies experience length contraction and time dilation. We shall see shortly that this new field or ether permeating space only has to do three things: endow all particles a mass dependent on their absolute velocity through it ${ }^{*}$, limit all particles from exceeding light speed and limit light speed.

\subsection{The reason for Constancy of Light Speed}

To the last point the photon, counter-intuitively, is seen to have an infinite effective mass (but not momentum $E=c p$ [2-3] and eqn. 17) centred on light speed, that prevents it from travelling at any other speed. This is similar to the way electrons behave in a semi-conductor in certain directions that forbade movement, due to the surrounding potentials. No-one is suggesting that the electron in this case actually has infinite mass or momentum in the conventional sense but more in the incremental differential sense, such as an electronic engineer might use to describe a non-linear resistance. For example a current source over a finite range of voltages resists changes to the current, so over a short range it can be said to have (near, in a real device) infinite resistance.

\footnotetext{
* This is directly related to the velocity two bodies perceive in relative motion between themselves by light speed limited measurements. If one of those bodies is in the rest frame, then it is indeed the same velocity.
}

\subsection{The General Absolute Transform}

The author in the earlier paper[12] then extended the argument to General Relativity to "flatten" space with differential local changes in the unit length and interval of time from both the effects of GR and SR[12]:

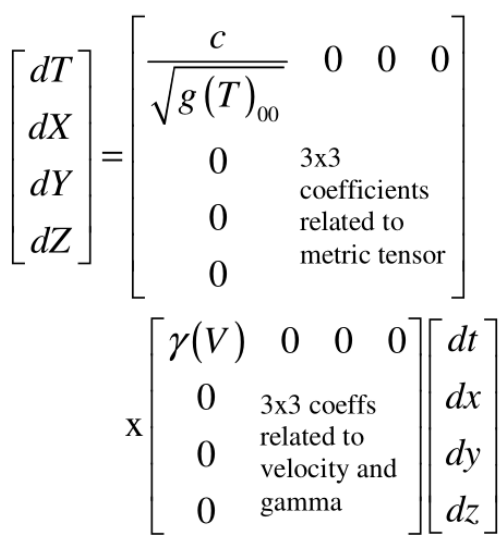

Thus the absolute co-ordinate system is Euclidian with no time dilation. If a gravitating source exists in the centre of the box, a rule moving from flat space at the extremities of the box (or a rule made from a light signal travelling for a set time) would contract and time would dilate in the field or from the motion such that the "proper" summed distance, measured by this travelling ruler, could be compared to the unchanging Euclidian grid.

What we are saying is that at absolute rest and away from a gravitating source, that unit time and length have a default value. In the first case regarding Special Relativity, motion through the ether field sets the length contraction and time dilation. In the second case with General Relativity, the stress-energy tensor modifies the ether field to cause extra length contraction and time dilation.

\subsection{Time dilation is explained by increase of effective mass}

The total time dilation is extracted from eqn. 20 by the relation of $\mathrm{dT}$ to $\mathrm{dt}$,

$$
d T=\frac{c}{\sqrt{g(T)_{00}}} \cdot \frac{1}{\sqrt{1-\frac{V^{2}}{c^{2}}}} \cdot d t
$$

Special Relativity prescribes the increase in mass of a moving body thus:

$$
m^{\prime}=\frac{m}{\sqrt{1-\frac{V^{2}}{c^{2}}}}
$$

We can contrive a moving clock based on simple harmonic motion (SHM) of a mass in a plane 
perpendicular (y axis) to the velocity (x axis). The Principle of Relativity states that we couldn't determine our state of uniform motion by any local measurement alone, so what we analyse with this clock is of general applicability, much as Einstein's moving light clock is not a result peculiar to light.

The SHM problem is set up thus:

$$
\begin{aligned}
& m \frac{d}{d t}\left(\frac{d y}{d t}\right)=-k y \\
& \frac{d^{2} Y}{d T^{2}}=-\frac{1}{\gamma^{2}} \frac{k}{m} Y
\end{aligned}
$$

In the last step, a transform to absolute coordinates (eqn. 19) is applied and the familiar solution yields:

$$
T_{p}=2 \pi \sqrt{\frac{m \gamma^{2}}{k}}
$$

The increase in mass of the moving clock would, from the perspective of the observer in frame [T, XYZ] seem to slow it by the same form as eqn. 22, in at least the gamma component of eqn. 21 , that is:

$$
T_{p}=\frac{1}{\sqrt{1-\frac{V^{2}}{c^{2}}}} \cdot t_{p}
$$

Where $t_{P}$ is the period in the moving frame and $T_{P}$, the period view from the absolute frame.

Using a simple relation between the relativistic momentum and the relativistic energy:

$$
E=\frac{m c^{2}}{\sqrt{1-\frac{v^{2}}{c^{2}}}}
$$

We argue that this mass increase is from the energy of interaction of the moving body with the ether. That is, if:

$$
E=\frac{p c^{2}}{v}
$$

The effective mass due to the relativistic energy can be found by noting (from eqn. 13),

$$
\begin{gathered}
\frac{\partial^{2} E}{\partial p^{2}}=\frac{\partial v}{\partial p} \\
\Rightarrow \frac{\partial^{2} E}{\partial p^{2}}=c^{2} \frac{\partial}{\partial p}\left(\frac{p}{E}\right)
\end{gathered}
$$

Thus we can regard "free-space" as being permeated by a potential varying as eqn. 26 , which leads to the relativistic mass, eqn. 22 and that is sufficient to account for "time dilation".

\subsection{Time dilation in General Relativity}

Consider the Schwartzchild metric[15] which is a good analytical example of a solution to the EFE:

$$
c^{2} d \tau^{2}=\left(1-\frac{r_{s}}{r}\right) c^{2} d t^{2}-\left(1-\frac{r_{s}}{r}\right)^{-1} d r^{2}-r^{2}\left(d \theta^{2}+\sin ^{2} \theta d \varphi^{2}\right)
$$

The $\mathrm{g}_{00}$ component shows how gravity slows time:

$$
g_{00}=\sqrt{1-\frac{2 G M}{c^{2} r}}
$$

The conclusion is that the modification of the local ether field by the local gravity field (eqn. 21) has the same form as eqn. 22 and acts to increase the mass still further.

In conclusion, we state that it is not correct to speak of time actually dilating; it is solely due to the inertia of mechanical components of a clock becoming more massive or in the case of a light clock, the constant speed of light.

\subsection{Length contraction effect is explained by increase of effective mass too}

Leptons and quarks are the basic components of matter in the Standard Model; they are believed to be point-like or in the least, extremely small. Matter is characterised by static fields, chemical bonds and the nuclear forces. All of these forces are understood to be based on the exchange of particles - photons for the static electrical forces, electrons for chemical bonds and $\mathrm{W}, \mathrm{Z}$ and gluons for the nuclear forces. These exchange processes can be represented quickly, for argument, by a toy model of a two state quantum system to illustrate a key point[5] about the binding energy and the wave mechanics.

The probability amplitude for a wave to move from one location to another by the wave equation (neglecting the variation with time) is:

$$
\left\langle\mathbf{r}_{2} \mid \mathbf{r}_{1}\right\rangle_{\text {bound }} \propto \frac{e^{i \frac{\mathbf{k} \cdot \mathbf{r}_{12}}{\hbar}}}{\mathbf{r}_{12}}
$$

The analysis of a bound state shows that the exchanged particle has negative energy. The nonrelativistic form of the kinetic energy immediately shows that the momentum must be imaginary: 


$$
p=\sqrt{2 E m}
$$

In the relativistic domain (from eqn. 10), the momentum is imaginary from the large rest mass of the exchanged particle:

$$
p=\frac{1}{c} \sqrt{E^{2}-m^{2} c^{4}}
$$

Thus in eqn. 31 the amplitude variation will decay with distance (an evanescent wave) and on substituting the momentum for wavenumber we obtain:

$$
\left\langle\mathbf{r}_{2} \mid \mathbf{r}_{1}\right\rangle_{\text {bound }} \propto \frac{e^{-\mathbf{p} \cdot \mathbf{r}_{12}}}{\mathbf{r}_{12}}
$$

If we adopt the co-ordinate system of eqn. 19 we can transform eqn. 34 to the view point from absolute space which is represented in capitalised co-ordinates:

$$
\begin{gathered}
\left\langle\mathbf{R}_{2} \mid \mathbf{R}_{1}\right\rangle_{\text {bound }} \propto \frac{e^{\sqrt{1-\frac{m \mathbf{V}}{c^{2}}} \cdot \mathbf{r}_{12}} \sqrt{1-\frac{V^{2}}{c^{2}}}}{\mathbf{r}_{12}} \\
\Rightarrow \frac{e^{-\frac{m \mathbf{V}}{\sqrt{1-\frac{V^{2}}{c^{2}}} \cdot \mathbf{R}_{12}}}}{\mathbf{R}_{12}}
\end{gathered}
$$

Thus the reason for length contraction becomes apparent: space itself isn't contracting the bond lengths associated with point masses and their exchanging point particles shortens in the direction of motion by the increase in mass of the exchanging particle and the consequent shorter decay of the evanescent wave as it moves through the ether.

Comparing eqn. 34 and eqn. 35 we can see they are of the same form but the frame at absolute rest perceives the exponential term to decay more quickly with distance. For instance eqn. 34 tells us that most of the probability distribution is contained in the distance with p. $\mathrm{r}_{12}=5$ (better than $99 \%$ of it, $\mathrm{e}^{-5}$ ). Viewed from the absolute frame (eqn. 35) and considering $\mathrm{p}$ to be the same, that distance has shrunk by a factor of gamma. We can say mysteriously that space contracts with motion or say that the true reason is that the mass has increased,

$$
d X=\frac{m^{\prime}}{m} d x
$$

With,

$$
m^{\prime}=\frac{m}{\sqrt{1-\frac{v^{2}}{c^{2}}}}
$$

Such that,

$$
d X=\frac{1}{\sqrt{1-\frac{v^{2}}{c^{2}}}} d x
$$

For long ranged forces, the ultra-relativistic case of zero photon mass, the dependency is primarily in the $1 / \mathrm{r}$ term. Viewed in the absolute frame, the dependency is $1 / \mathrm{R}$, which can be transformed into the moving frame by the following procedure:

$$
\frac{1}{R^{2}} \mathbf{R} \cdot \frac{\mathbf{p}}{|P|} \equiv \frac{1}{\gamma^{2} r^{2}} \gamma r \cdot \frac{\mathbf{p}}{|P|}
$$

Where $\mathrm{P}$ is the momentum of the photon in the absolute frame and $\mathrm{p}$ is the momentum in the moving frame. Thus the absolute frame observes the moving frame to be length contracted.

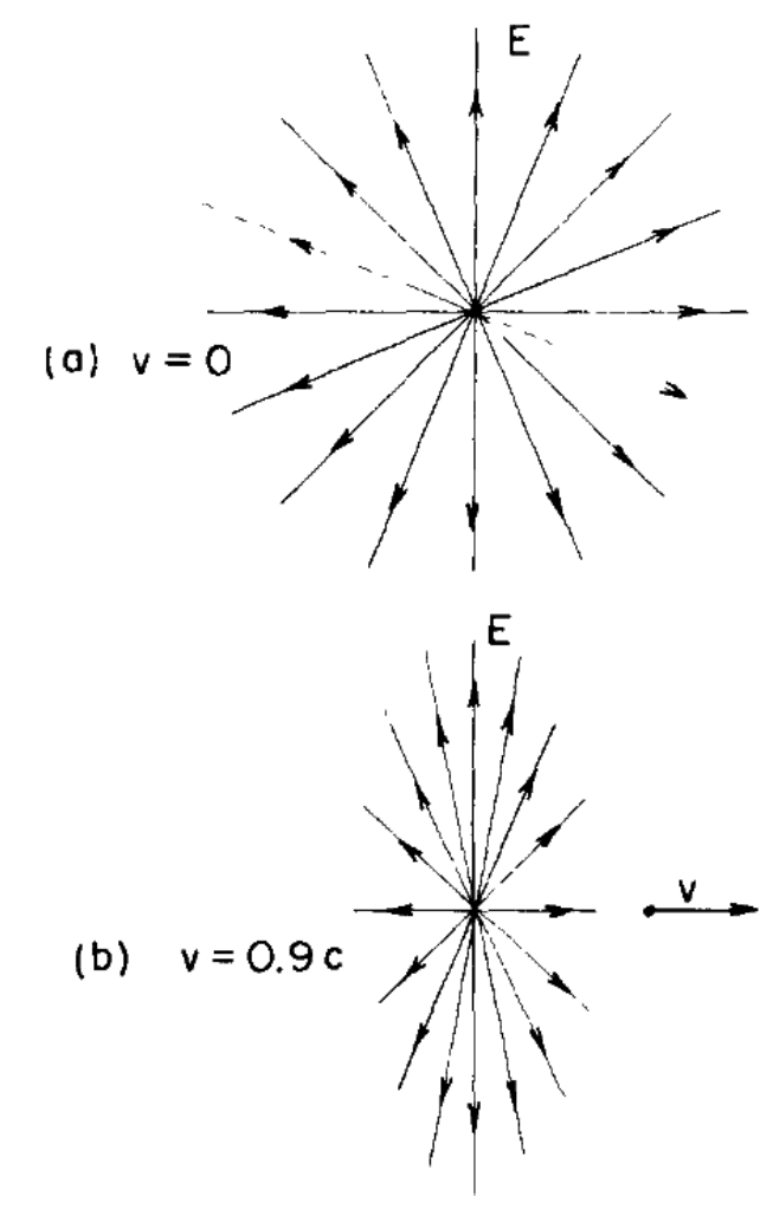

Figure 2 - (from Feynman[5]) The bunching of field lines in the direction of motion of the charge. 
On the macroscopic level we know that time dilation is a real effect, as we can tell from travelling twin experiments with atomic clocks. However it is not so easy to convince oneself that length contraction is a real effect with the classic Barn and Pole thought experiment! We couldn't build anything large enough and move it fast enough, to directly infer length contraction or tidal forces arising from parts of the apparatus not being in the same frame. There isn't a similar travelling twin scenario, as length doesn't monotonically increase like time - we can't compare the two macroscopic cases. Observation of synchrotron radiation, the bunching of field lines, the design of accelerators, all show the truth of length contraction but we believe here that it has been given an absolute physical basis.

\subsection{Length contraction with motion non-collinear} to the axis and General Relativity

In considering the case of general motion in any direction, expansion of the spatial element of the second matrix (the "gamma matrix") in eqn. 20 is informative[12]:

$$
\left[\begin{array}{l}
d X \\
d Y \\
d Z
\end{array}\right]=\left[\begin{array}{ccc}
1+(\gamma-1) \frac{V_{x}^{2}}{V^{2}} & (\gamma-1) \frac{V_{x} V_{y}}{V^{2}} & (\gamma-1) \frac{V_{x} V_{z}}{V^{2}} \\
(\gamma-1) \frac{V_{x} V_{y}}{V^{2}} & 1+(\gamma-1) \frac{V_{y}^{2}}{V^{2}} & (\gamma-1) \frac{V_{y} V_{z}}{V^{2}} \\
(\gamma-1) \frac{V_{x} V_{z}}{V^{2}} & (\gamma-1) \frac{V_{y} V_{z}}{V^{2}} & 1+(\gamma-1) \frac{V_{z}^{2}}{V^{2}}
\end{array}\right]\left[\begin{array}{l}
d x \\
d y \\
d z
\end{array}\right] \text { eqn. 38 }
$$

This matrix is obtained by the tensor rotation of the simple case of motion along one axis through the three Euler angles:

And

$$
\lambda^{\prime}=R \lambda^{T} R^{T}
$$

$$
R=R_{x} R_{y} R_{z}
$$

Which on generalising eqn. 36 gives a tensorial nature to the mass in eqn. 35 ,

$$
\left[\begin{array}{l}
d X \\
d Y \\
d Z
\end{array}\right]=\frac{1}{m}\left[\begin{array}{lll}
m_{11} & m_{12} & m_{13} \\
m_{21} & m_{22} & m_{23} \\
m_{31} & m_{32} & m_{33}
\end{array}\right]\left[\begin{array}{l}
d x \\
d y \\
d z
\end{array}\right] \text { eqn. } 41
$$

Anisotropic effective mass is already a feature of semiconductor physics (eqn. 17). It is most generally expressed as a tensor reflecting its spatial variation:

$$
M_{i j}=\frac{\hbar^{2}}{\frac{\partial^{2} E}{\partial k_{i} \partial k_{j}}}
$$

Relativity and the matrix of apparent effective masses formed (eqn. 36 and eqn. 41) gets the same treatment for General Relativity. The $3 \times 3 \mathrm{sub}-$ matrix in eqn. 20 relate to the metric tensor and this is multiplied by the gamma matrix discussed previously.

\section{Conclusion}

This paper has sought to put relativistic effects on a materialistic objective footing. Existing physics is put on a passive (at present) backdrop of an Ether field that explains relativistic effects of time dilation and length contraction by mass gains of particles. The analogy and formalism is similar to the use of effective mass in semiconductor physics where particle interaction with the lattice is responsible for the effective mass.

In the case of time dilation, this can be explained by considering the case of mass increase in something as simple (and general) as simple harmonic motion. A "light clock" is explained by the finite speed limit of light, which is derived from the photon's interaction with the ether field.

It is believed that kinetic energy is placed on a similar footing to potential energy, where velocity squared represents the displacement in the field. It is believed that all mass derives from the effective mass against this ether backdrop.

Further to the idea of General Relativity, with the metric and gamma providing a backdrop for the physics of the effective mass coupling to the standard model, an attempt has not yet been made to show the dynamics of this ether proxy for GR and hence the effect of the physics overlain onto it (i.e. the stress-tensor modifies the metric).

The treatment is nascent but differs from other modern ether theories by generating Lorentz invariance from an underlying flat space time. This comes as the result of the author's earlier inquiry into apparent faster-than-light communication by a protocol to send classical data over a Bell channel.

The Ether is a field with energy (it may too, as a proxy, explain anomalies in the definition of gravitational energy) and this must be subject to quantisation. However, the underlying premise of flat space may remove some of the traditional difficulties associated with forming quantum theories of gravity. As a proxy, it may offer another route. Also the situation with covariant theories doesn't change; any result so derived automatically applies to the rest frame but with the added bonus that the result can be considered absolute and a material property of absolute space. 
In all, for progress in other fields, such as advanced propulsion, it is hoped that some of the mystery is removed surrounding the speed of light and Lorentz invariance by attributing them to physical effects. The desire is, that the coupling from the Ether might be modified and lead to new physics on the other side of the barrier, much as surmounting the sound barrier lead to a new era of aerodynamics.

\section{$\underline{\text { References }}$}

1. The Oxford Handbook of the History of Physics, ed. J.Z.B.R. Fox. 2013: Oxford University Press.

2. Feynman, L., Sands, The Feynman Lectures on Physics. Addison-Wesley, Reading, Massachusetts. Vol. Vol.1, Vol. 2 1989. 14.7, 15.1-6, 35.4

3. Jackson, J.D., Classical Electrodynamics. 2nd ed. 1975: Wiley.

4. Misner, C.; Thorne, K.; Wheeler, J., Gravitation. 21st ed. 1998: W. H. Freeman and Co.

5. Feynman, Leighton, Sands, The Feynman Lectures on Physics. Addison-Wesley, Reading, Massachusetts. Vol. 1, 3. 1989.

6. Landau, Lifshitz, A Course in Theoretical Physics: Quantum Mechanics. Vol. 3. 1982: Butterworth-Heinemann.

7. Peleg, Yoav; Pnini, Reuven; Zaarur Elyahu, Schaum's Outlines, Quantum Mechanics. 1998: McGraw-Hill.

8. Landau, Lifshitz, A Course in Theoretical Physics: Quantum Electrodynamics. Butterworth-Heinemann. Vol. 4. 1984.

9. K. P. Sinha, C. Sivaram, E. C. G. Sudarshan, Aether as a Superfluid State of Particle-Antiparticle Pairs. Found. Phys., 1976. 6.

10. K. P. Sinha, E. C. G. Sudarshan, The Superfluid as a Source of All Interactions. Found. Phys., 1978. 8.

11. Cornwall, R.O., Secure Quantum Communication and Superluminal Signalling on the Bell Channel. Infinite Energy, 2006. 69

(http://vixra.org/abs/1311.0074).
12. Cornwall, R.O., Is the Consequence of Superluminal Signalling to Physics Absolute Motion Through an Ether? Infinite Energy, 2010. 98 (http://vixra.org/abs/1311.0075).

13. Zbinden H., Gisin N., Testing the speed of 'spooky action at a distance'. Nature, 2008. 454.

14. Dopfer, Birgit, Two experiments on the interference of two-photon states. $\mathrm{PhD}$ Thesis, University of Innsbruck, 1998.

15. Landau, Lifshitz, A Course in Theoretical Physics: The Classical Theory of Fields. Vol. 2. 1982: Butterworth-Heinemann. 